\title{
Rheology of non-Newtonian suspensions of fly ash: effect of concentration, yield stress and hydrodynamic interactions
}

\author{
B. Bournonville, A. Nzihou* \\ Laboratoire de Génie des Solides Divisés, UMR-CNRS 2392, Centre Energétique et Environnement, Ecole des Mines d'Albi-Carmaux, \\ Campus Jarlard Route de Teillet, 81013, Albi CT cedex 09, France
}

\begin{abstract}
Two different water-washed municipal solid waste (MSW) incinerator fly ashes were investigated. The rheological behaviour of these fly ashes in aqueous suspensions was studied using a parallel plate rheometer. Shear-thinning behaviour was observed in one sample whereas thixotropic behaviour was found in the other. Viscosity depends on both the volume fraction of solids in the suspension and on the yield stress. The data could be well correlated to a model we proposed which takes into account the volume fraction of solid and the shear rate. The comparison with various models of the literature is discussed.

The hydrodynamic theory of particle movement in non-Newtonian fluids is discussed, focusing on inertia and viscous effects. Two modified Reynolds numbers involving respectively the shear rate and the yield stress, and with the shear rate and the volume fraction of solids, are proposed and discussed. The flow regime in the investigated experimental conditions is laminar and governed by hydrodynamic interactions. The results also show that inertia cannot be neglected.
\end{abstract}

Keywords: Rheology; Concentrated suspensions; Shear-thinning; Reynolds number; Interactions

\section{Introduction}

The incineration of municipal waste generates fly ash residues which contain soluble chlorides and heavy metal ions [1-3]. Currently, the fly ash is landfilled after a cement solidification step. A new treatment uses phosphoric acid to neutralise fly ash basicity and to form metal phosphates, which are insoluble in natural environments. We have used water-washed fly ashes treated with phosphoric acid to produce heat stable mineral species, which trapped metal ions in insoluble form. Such products may be landfilled or reused as mineral fillers. The process (NEUTREC ${ }^{\circledR}$ ) is composed of the following steps: first, the fly ash is washed and filtered to extract soluble salts (mostly chlorides) before chemical treatment with phosphoric acid. Second, thermal treatment dries the residues and transforms the solids into a stable mineral species.

To define optimum conditions for this new process, the rheology of washed fly ash suspensions must be investigated with and without phosphoric acid addition. We have devel\footnotetext{
30-99.

* Corresponding author. Tel.: +33-5-63-49-32-22; fax: +33-5-63-49-

E-mail address: nzihou@enstimac.fr (A. Nzihou).
}

oped a model that describes the experimental viscosity data in terms of both solid volume fraction and shear rate. The flow regime is discussed using a modified Reynolds number. This contributes to a better understanding of reactor hydrodynamics in laboratory and pilot scale plants. We report here on the rheological properties of two different fly ashes before phosphoric acid treatment.

Theoretical approach to the rheology. Rheology is defined as the science of deformation and flow of matter in response to the application of mechanical force.

Simple shear deformation is shown in Fig. 1. The upper plate can move parallel to the lower fixed plate under the influence of an applied force $F$. A thin slice of material of constant thickness is trapped between the two plates and adheres to both. The area of upper plate is $S$. The shear stress $\tau$ is defined as force per unit area Eq. (1):

$\tau(\mathrm{Pa})=\frac{\Delta F}{\Delta S}$

The amount of deformation is known as the shear strain $\gamma$ and defined by:

$\gamma=\frac{\Delta x}{\Delta y}$ 


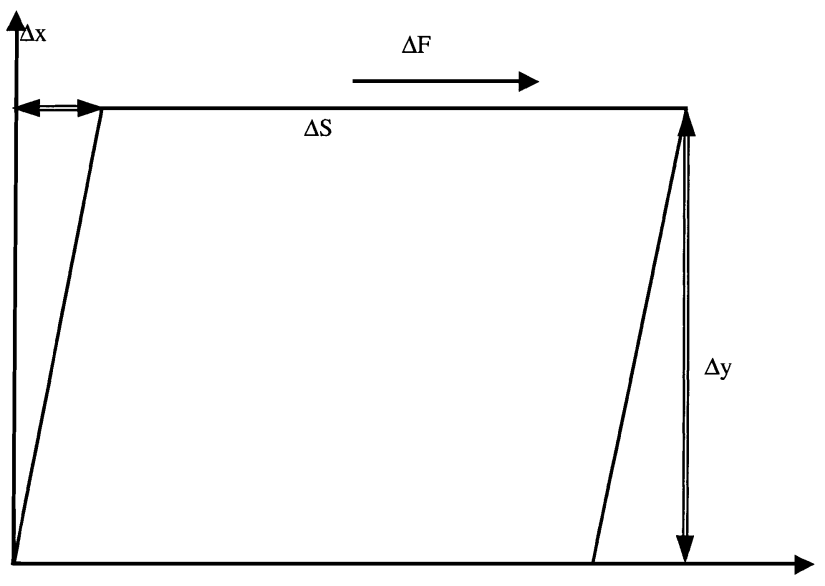

Fig. 1. Single shear strain.

When force is applied, the upper plate moves at a constant velocity as long as the force is applied. In an analogous fashion, a shear strain rate, usually known as the shear rate $\gamma$ is defined as follows:

$\dot{\gamma}\left(\mathrm{s}^{-1}\right)=\frac{\partial \gamma}{\partial t}$

In steady-state regime, the shear rate is proportional to the stress:

$\tau=\mu \dot{\gamma}$

where the constant of proportionality $\mu$ is the shear viscosity or the viscosity $(\mathrm{Pa} \cdot \mathrm{s})$.

\section{Materials and methods}

Fly ashes used in this study were taken from two different municipal solid waste (MSW) incinerators and labelled S and $\mathrm{O}$. These fly ashes contain water-soluble salts that were extracted by suspension in water in a laboratory reactor. The liquid phase extract was filtered and evaporated, and the water-soluble contents were determined. The solid fraction in the aqueous suspension without soluble salts (mass fraction of solid, $X$ from $30 \%$ to $55 \%$ ) was used as sample for the experiments described in this paper.

\subsection{Characterization}

Particle size distribution (mean size) was determined with a Mastersizer laser granulometer (Malvern). Results obtained show a common mean size of $28 \mu \mathrm{m}$ for each of fly ashes $\mathrm{O}$ and S. Because of the difficulty to disperse the fly ash particle during the size measurement, the mean size obtained may correspond to aggregates. Densities $\left(\rho_{\mathrm{p}}\right)$ of $2460 \mathrm{~kg} / \mathrm{m}^{3}$ were obtained for $\mathrm{O}$ and $2720 \mathrm{~kg} / \mathrm{m}^{3}$ for $\mathrm{S}$ with a Micromeritics 1330 gas pycnometer. The specific surface areas determined by the BET method in a Gemini Micromeritics instrument are $15.8 \mathrm{~m}^{2} / \mathrm{g}$ for $\mathrm{O}$ and $10.8 \mathrm{~m}^{2} / \mathrm{g}$ for $\mathrm{S}$.
X-ray fluorescence measurements were made with a Philips SEM with energy dispersive analysis for major elements (calculated in oxide form) and Inductively Coupled Plasma (ICP) for minor elements. Fly ash $\mathrm{S}$ is composed as follows: $\mathrm{Al}_{2} \mathrm{O}_{3}(9 \%), \mathrm{CaO}(25 \%), \mathrm{Fe}_{2} \mathrm{O}_{3}(8 \%), \mathrm{SiO}_{2}(18 \%)$, $\mathrm{SO}_{4}$ (20\%), $\mathrm{Cr}$ (1126 ppm), $\mathrm{Cu}$ (2552 ppm), $\mathrm{Pb}$ (12500 ppm) and $\mathrm{Zn}(28500 \mathrm{ppm})$; composition of fly ash $\mathrm{O}: \mathrm{Al}_{2} \mathrm{O}_{3}(17 \%)$, $\mathrm{CaO}(25 \%), \mathrm{Fe}_{2} \mathrm{O}_{3}(2 \%), \mathrm{SiO}_{2}(28 \%), \mathrm{SO}_{4}(2 \%), \mathrm{Cr}(513$ ppm), $\mathrm{Cu}$ (1022 ppm), Pb (3731 ppm) and Zn (12417 ppm).

\subsection{Rheology measurements}

Rheological measurements were carried out with a RS150 Rheostress rheometer (Haake) operating as a controlled stress instrument. The measuring system consists of two parts (Fig. 2). One is the fixed member and the second is attached to the driving motor spindle where it is locked in position by means of the draw rod. The geometry is fixed by the moving member (rotor). We have used the roughened geometry parallel plate with a $2-\mathrm{cm}$ diameter. The gap size for the suspensions is generally set at least 10 times higher than the largest particle size. The gap between parallel rotating plan and the fixed plate is $500 \mu \mathrm{m}$. The specifications of the rheometer are: torque variation from $0.5 \times 10^{-4}$ to $150 \mathrm{mNm}$, shear rate from $10^{-7}$ to $1920 \mathrm{~s}^{-1}$. The experimental conditions were as follows: shear rate from 0.01 to $8 \mathrm{~s}^{-1}$, time $180 \mathrm{~s}$ and temperature $23{ }^{\circ} \mathrm{C}$.

\section{Results and discussions}

\subsection{Rheological behaviour of the fly ash suspension}

The rheological behaviour of suspensions of the two ashes was investigated at $23{ }^{\circ} \mathrm{C}$. In the curves of Fig. $3 \mathrm{a}$ and $\mathrm{b}$, the viscosity $\mu$ of each fly ash is found to decrease with increase in shear rate, leading to what is now generally called "shear-thinning" or "pseudoplastic" behaviour.

The shear-thinning behaviour observed in the viscosity curves is confirmed by the shape of the rheogram $\tau-\dot{\gamma}$ of Fig. $4 \mathrm{a}$ and $\mathrm{b}$.

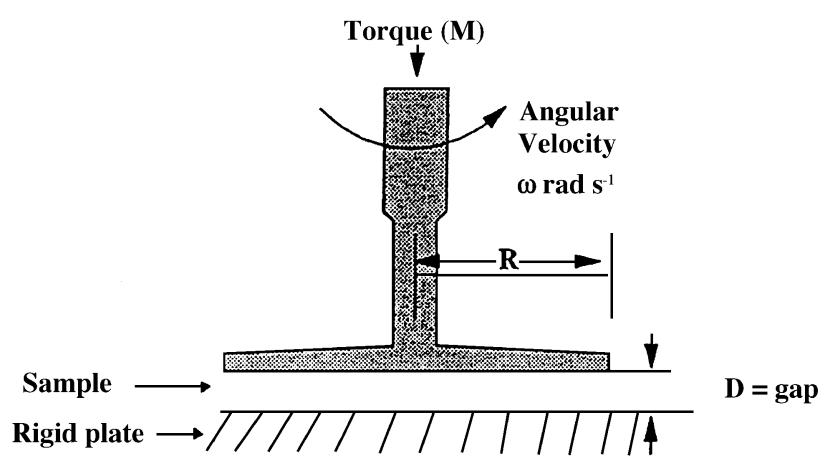

Fig. 2. Basic geometry for plate-and-plate flow. 

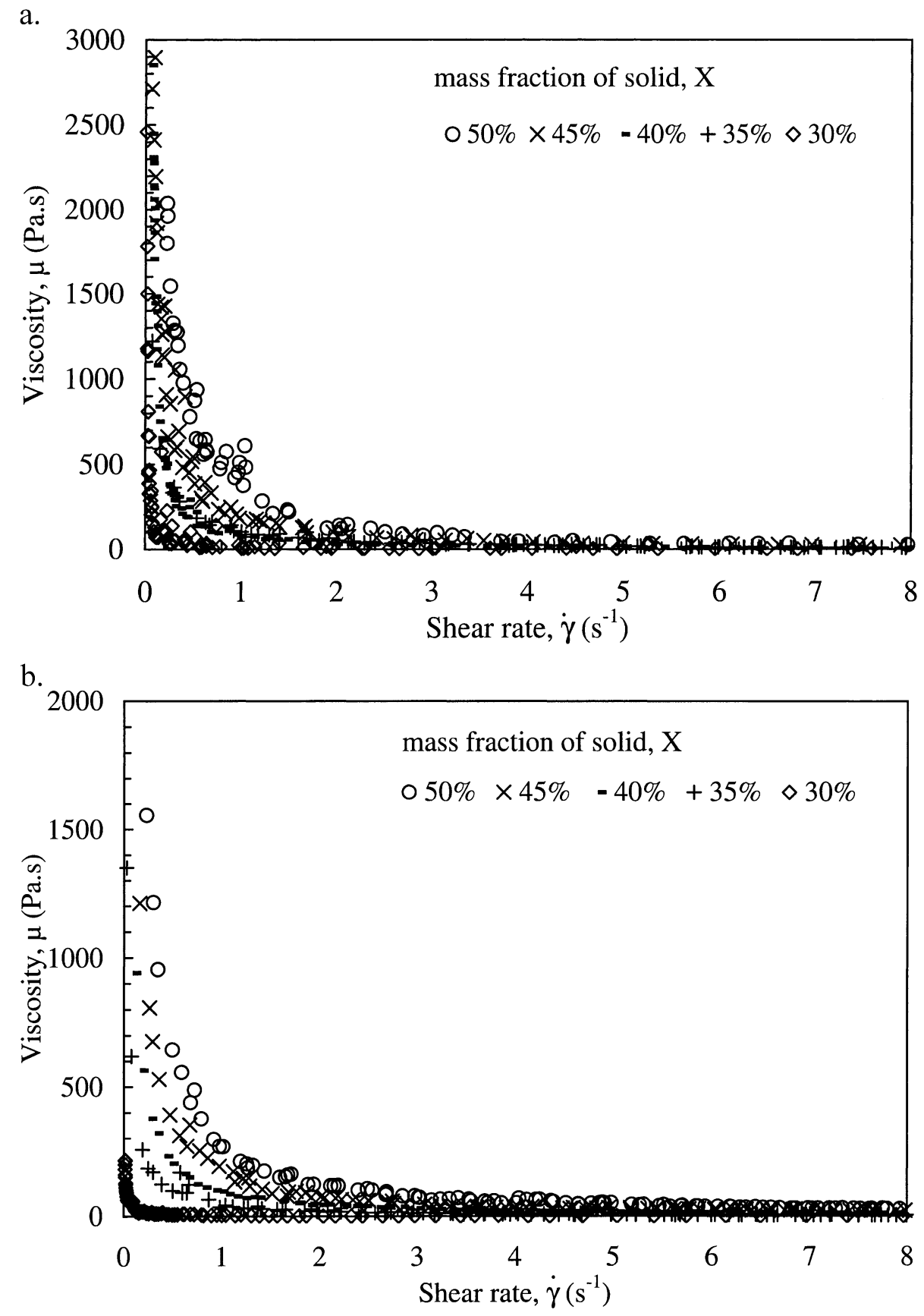

Fig. 3. (a) Shear viscosity versus shear rate for O. (b) Shear viscosity versus shear rate for S.

The curves of Fig. $3 a$ and $b$ also show that fly ash $\mathrm{O}$ is more viscous than fly ash S. Generally, in shear-thinning behaviour, the curves indicate that, in the case of very low shear rates (or stresses), the viscosity is constant, while in the case of high shear rates (or stresses), the viscosity is again constant, but lower. These two extremes correspond to first and second Newtonian regions. The described viscosity behaviour can be correlated to the Cross model that predicts the shape of the general flow curve (Fig. 5) [4,5]:

$\frac{\mu-\mu_{\infty}}{\mu_{0}-\mu_{\infty}}=\frac{1}{1+(K \dot{\gamma})^{m}}$ where $\mu_{0}$ and $\mu_{\infty}$ refer to asymptotic values of viscosity at very low and high shear rates respectively. $\mathrm{K}$ is a constant parameter with the dimension of time and $\mathrm{m}$ is a dimensionless constant.

For the results presented in Fig. 3, which are graphs of viscosity against shear rate for several mass fractions of solids in the suspension, no first Newtonian region is observed. This region should appear at very low shear rate as shown in the literature [4]. The curves only exhibit the second Newtonian region. This corresponds to infinite shear viscosity and is observed in both ashes $\mathrm{S}$ and $\mathrm{O}$. 

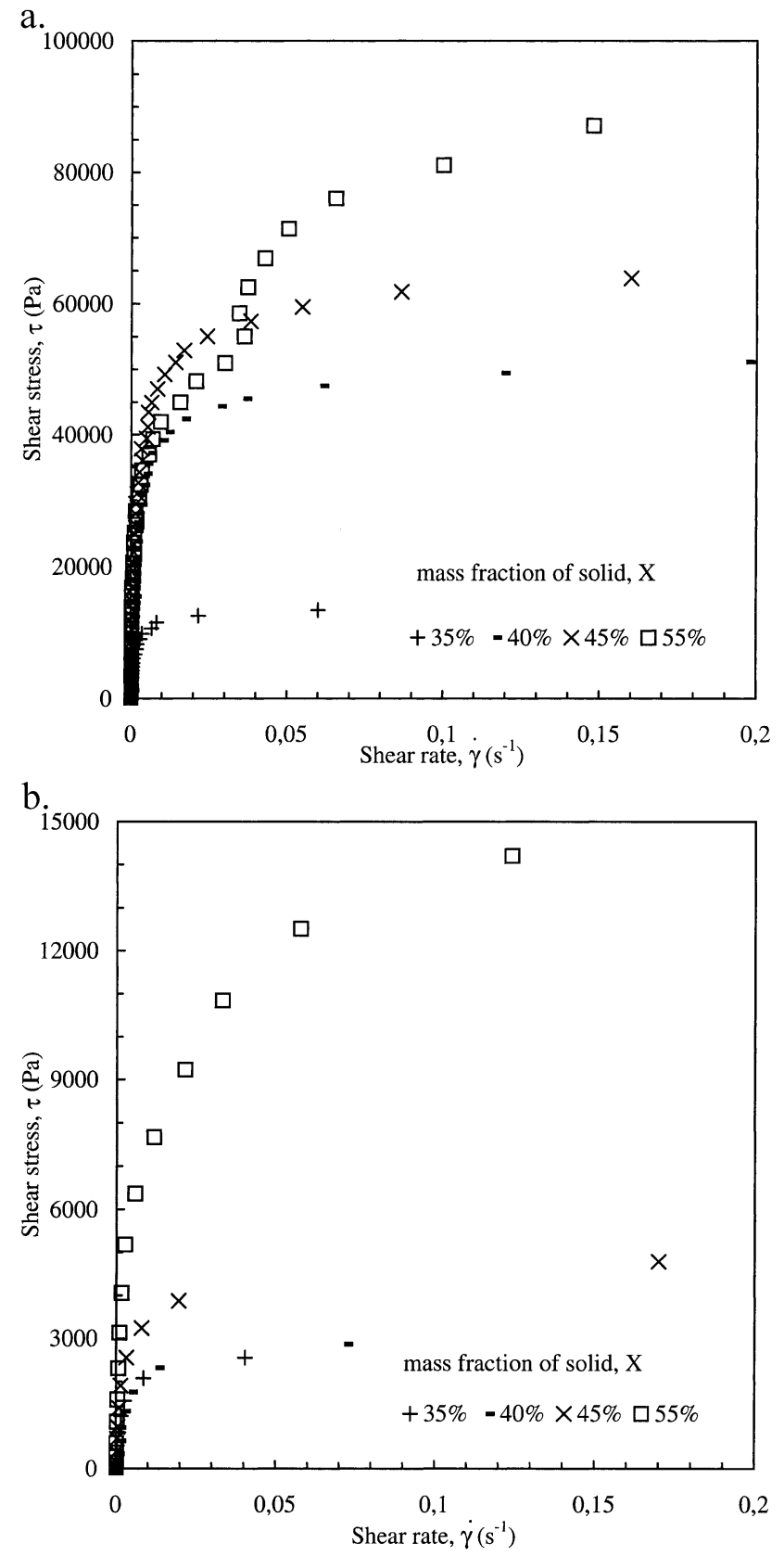

Fig. 4. (a) Flow curves of the fly ash O. (b) Flow curves of the fly ash S.

\subsection{Thixotropic effect}

Ashes $\mathrm{O}$ and $\mathrm{S}$ present a similar shear plastic behaviour at low shear rate (Fig. $4 \mathrm{a}$ and $\mathrm{b}$ ). Ash $\mathrm{S}$ shows thixotropic behaviour (Fig. 6) at high shear rate. An apparent yield stress is observed for each fly ash (see Section 3.3 for the discussion).

The reviews of Mewis [6,7] provided physical explanations for thixotropic phenomena along with an exhaustive, and critical, review of corresponding measurement methods. We shall only summarise these works and give some additional comments accounting for the results presented in this paper.
When the shear rate is steadily increased at a constant rate from zero to some maximum value and then decreased at the same rate to zero shear rate, a hysteresis loop as shown in Fig. 6 is observed. The position of this loop, its shape and the area within the loop depend on the increase and decrease rates of the shear rate as well as the past thermal and shear history of the fly ash.

The fluid viscosity is time-dependent. The apparent viscosity decreases with time of shear. The behaviour of these materials depends not only on the time of shear but also on the past shear and thermal history.

The difference between thixotropy and pseudoplastic behaviours observed with fly ashes $\mathrm{S}$ and $\mathrm{O}$ is thought to be related to the time of structural breakdown, which is finite and measurable for the thixotropic fluid, but very small and undetectable for the pseudoplastic fluid.

Nevertheless, as the results presented show, thixotropy usually occurs in circumstances where the suspension is shear-thinning in the sense that viscosity decreases with increasing shear rate, other things being equal [4,8]. It means that both fly ashes have the same basic shearthinning behaviour. In fact, gelification was observed under these conditions. The occurrence of thixotropy implies that flow history must be taken into account when making predictions of flow behaviour.

\subsection{Yield stress}

Another characteristic of the fly ashes is the apparent yield stress exhibited during the flow of the suspension. Suspensions often exhibit yield stress, as soon as the particle concentration is sufficiently large. Fig. 7 shows that the suspension yield stress seems to tend towards infinity when the solid concentration tends to a finite value. The reason is that bonds between particles may form a continuous network which breaks only when a minimum force is applied $[9,10]$. Direct frictional contact between particles is impossible in the suspension when the solid fraction is so large that particles are more or less packed against each other and forced to remain in contact. Within granular packing, there exists a specific pressure due to particle contacts and it is conceivable that a finite force is required to break the contact network.

Gelification observed can also explain the yield stress behaviour. In fact, it would also suggest a colloidal behaviour: for sufficiently small suspended particles and slow flows, various types of electrochemical (colloidal) forces can become larger than common hydrodynamic interactions. So colloidal effects are mainly Van der Waals forces, double-layer interactions and steric effects. The particularity of these forces is to give rise to interaction between particles at relatively long distances even at rest $[11,12]$.

Though it is difficult to determine the exact value of yield stress with a conventional rheometer like that used in this study, recent experimental results [4,13-15] tend to demonstrate that there exists a concentration threshold above 


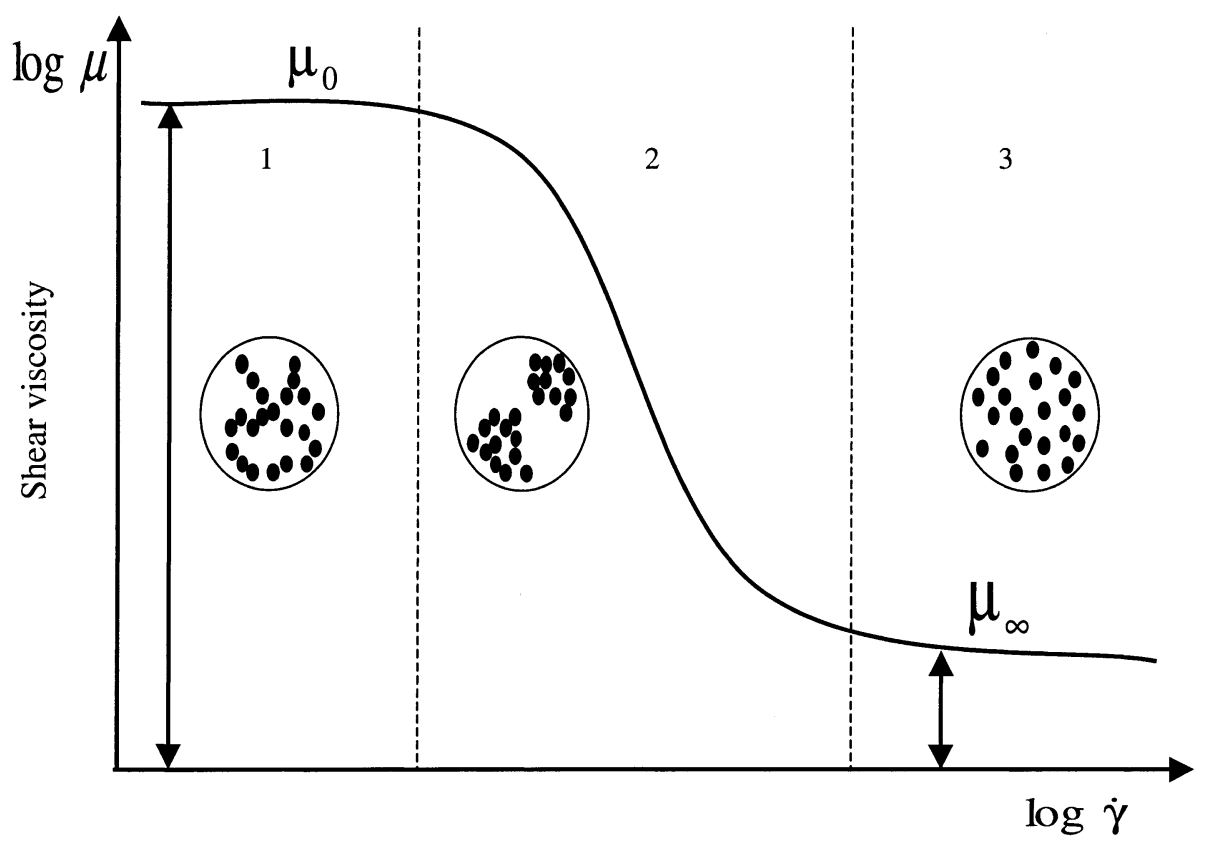

Fig. 5. Theoretical Cross model. 1-Percolating network, 2-suspension of clusters, 3-dispersed suspension [5].

which a concentrated force-free particle suspension exhibits yield stress. There is not yet a general agreement concerning the critical solid fraction value above which this yield stress appears. Our value of around $30 \%$ solids is different from the one suggested by Coussot and Piau [15] and by rough estimations from the results of Barnes et al. [4], 56\%; and from that deduced from Kytomaa and Prassad [13], 50\%. The difference observed could be due either to differences in composition or in the particular shear rate range investigated.

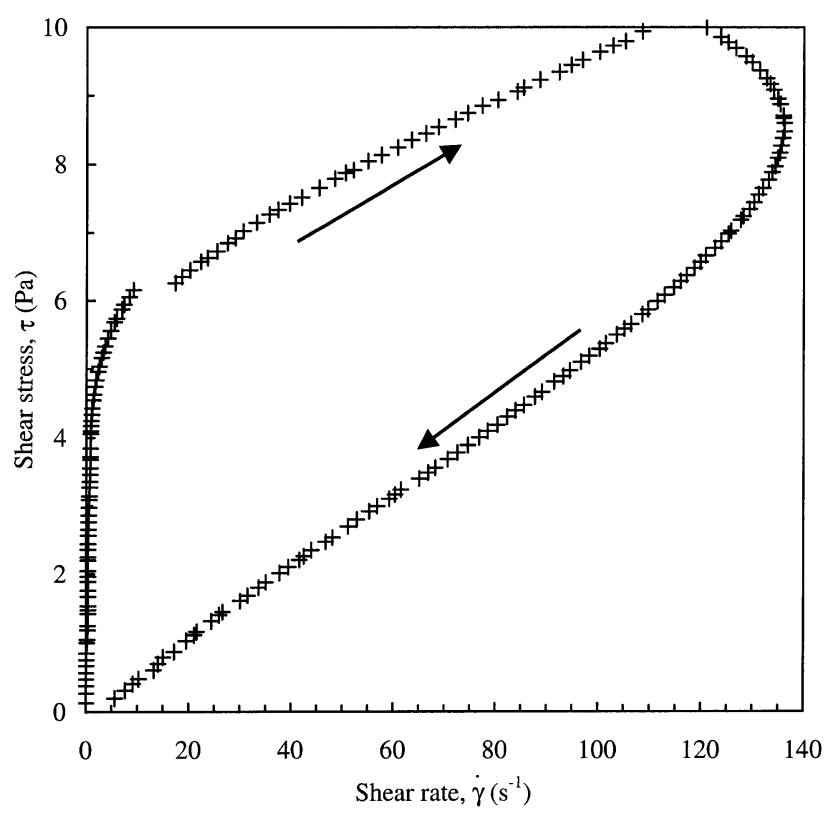

Fig. 6. Thixotropic behaviour of $\mathrm{S}$ fly ash at mass fraction $X=35 \%$.
This is analogous to the phenomenon of viscosity singularity and can be associated with the existence of a concentration threshold, i.e. a "maximum packing concentration", for which no deformation can take place without breakage or volume change [16]. The appearance yield stress above a certain solid fraction represents a sharp change in the physical properties of the suspension. The transition from a fluid to solid state as associated with this yield stress was for example compared to glass transition [17]. For concentrated suspensions, the reality might be more complex: depending

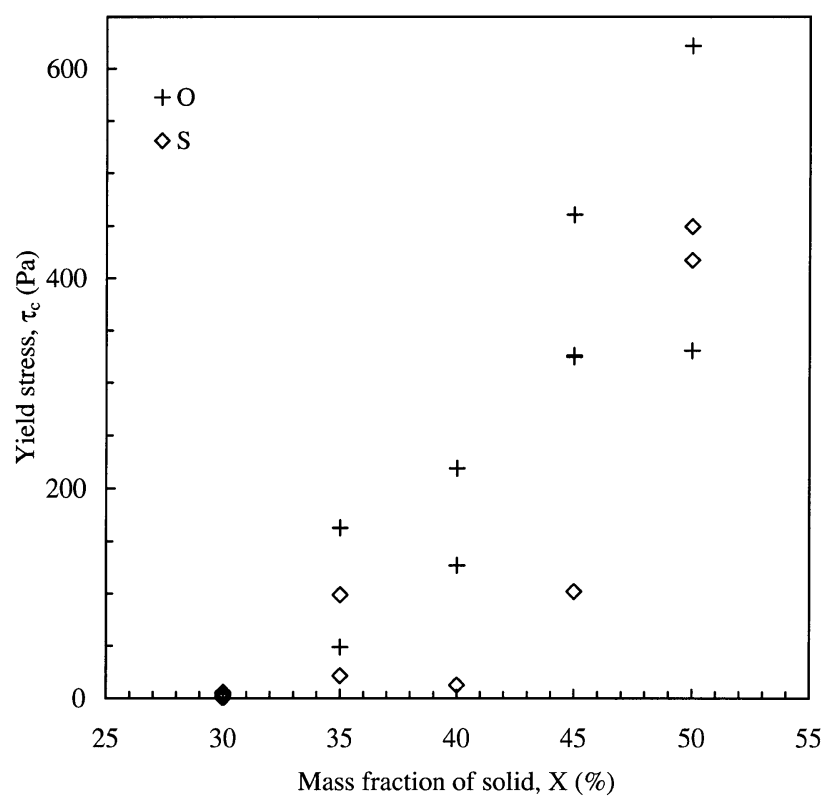

Fig. 7. Yield stress of fly ash suspension $\mathrm{O}$ and $\mathrm{S}$ as a function of mass solid fraction. 
on the average configuration, the predominant interaction type during flow (see Section 3.5 for the discussion) can either be direct contact or viscous dissipation within the interstitial fluid. For an increasing shear rate, there could be a progressive change from a "close configuration" within which particles may essentially interact via solid friction, to a "loose configuration" within which particles mainly interact hydrodynamically. Since these changes in configuration are not instantaneous, thixotropic effects may then be observed.

\subsection{Modeling of viscosity measurements}

Fig. $3 \mathrm{a}$ and $\mathrm{b}$ shows that the viscosity of the suspension increases with increasing solid mass fraction as usually observed for concentrated suspensions. However, two experimental limits were observed. The curve representing $\mu$ against $\dot{\gamma}$ at $X=55 \%$ shows erratic values for both $\mathrm{O}$ and $\mathrm{S}$ fly ashes, and is not presented here. High concentration during the measurement can explain these phenomena-the particle between the two parallel plates of the rheometer slipped and made the measurement difficult. This mass fraction of solids can be considered as a maximum mass fraction of solid in the suspension of fly ash. This value must be taken into account when choosing reactor operating conditions. Erroneous values are also observed at $X=30 \%$, which can be explained by the sedimentation observed in the suspension.

Many attempts have been made to describe the concentration dependence of viscosity [17-25]. In order to take into account the packing effect and the impossibility of having a concentration larger than the maximum packing fraction, it is conventional [18-20] to modify the above approach by using the ratio of solid fraction to maximum packing fraction $\phi_{\text {max }}$ instead of the simple solid volume fraction $\phi$ in the equations presented in the following section:

The Eiler [20] model is defined as

$\mu_{\mathrm{r}}=\left[1+1,25 \frac{\phi}{1-\frac{\phi}{\phi_{\max }}}\right]^{2}$

The Chong et al. [21] approach for polydispersed suspensions is:

$\mu_{\mathrm{r}}=\left[1+0,75 \frac{\frac{\phi}{\phi_{\max }}}{\left(1-\frac{\phi}{\phi_{\max }}\right)}\right]^{2}$

Krieger and Dougherty [22] proposes

$\mu_{\mathrm{r}}=\left(1-\frac{\phi}{\phi_{\max }}\right)^{-[\mu] \phi_{\max }}$ $[\mu]$ is the intrinsic viscosity, which is expected to be 2.5 for spheres.

In the Quemada model [17], the coefficient $[\mu]$ has been set to 2 ,

$\mu_{\mathrm{r}}=\left(1-\frac{\phi}{\phi_{\max }}\right)^{-2 \phi_{\max }}$

Nzihou et al. [23] proposed a model showing the dependence of viscosity on shear stress:

$\mu_{\mathrm{r}}=\left[1+A \frac{\frac{\phi}{\phi_{\max }}}{\left(1-\frac{\phi}{\phi_{\max }}\right)}\right]^{B}$,

$A=0.81$ and $B=0.5 \tau-1.3$

In these models, $\mu_{\mathrm{r}}$ is the reduced viscosity $\mu_{\mathrm{r}}=\mu / \mu_{\mathrm{s}}$ (with $\mu_{\mathrm{s}}$ continuous phase viscosity $=10^{-3} \mathrm{~Pa} \cdot \mathrm{s}$ in our study) [19] and $\phi$ is the volume fraction of particles in suspension. The volume fraction is defined as the ratio of fly ash volume to the suspension volume $V$ :

$\phi=\frac{V_{\mathrm{p}}}{V}=\frac{V_{\mathrm{p}}}{V_{\mathrm{p}}+V_{\mathrm{s}}}$

where $V_{\mathrm{s}}$ is the volume of the continuous phase

$\phi=\frac{\frac{X}{\rho_{\mathrm{p}}}}{\frac{X}{\rho_{\mathrm{p}}}+\frac{(1-X)}{\rho_{\mathrm{s}}}}$

where $X$ is the mass fraction of solid (weight percent) and $\rho_{\mathrm{s}}$ and $\rho_{\mathrm{p}}$, respectively, the water and particle density $\left(\mathrm{kg} / \mathrm{m}^{3}\right)$.

The correspondence between mass fraction and volume fraction is given in Table 1.

Eqs. (6)-(10) involve a parameter, $\phi_{\max }$, corresponding to the maximum random packing fraction of particles $\left(\phi_{\max }=0.62[4,20]\right)$. The results presented in Fig. 8 corresponding only to fly ash $\mathrm{S}$, show that the model proposed by Nzihou et al. gives a best correlation in comparison with other models [20-23]. Nevertheless, the difference between this model and the experimental data increases at high shear rate. The same result was obtained for fly ash $\mathrm{O}$ and is not reported here.

Table 1

Calculation of volume fraction of solids using the mass fraction

\begin{tabular}{lll}
\hline Mass fraction, $X(\%)$ & \multicolumn{2}{l}{ Volume fraction, $\Phi$} \\
\cline { 2 - 3 } & S fly ash & O fly ash \\
\hline 30 & 0.14 & 0.15 \\
35 & 0.17 & 0.18 \\
40 & 0.20 & 0.21 \\
45 & 0.23 & 0.25 \\
50 & 0.27 & 0.29 \\
55 & 0.31 & 0.33 \\
\hline
\end{tabular}



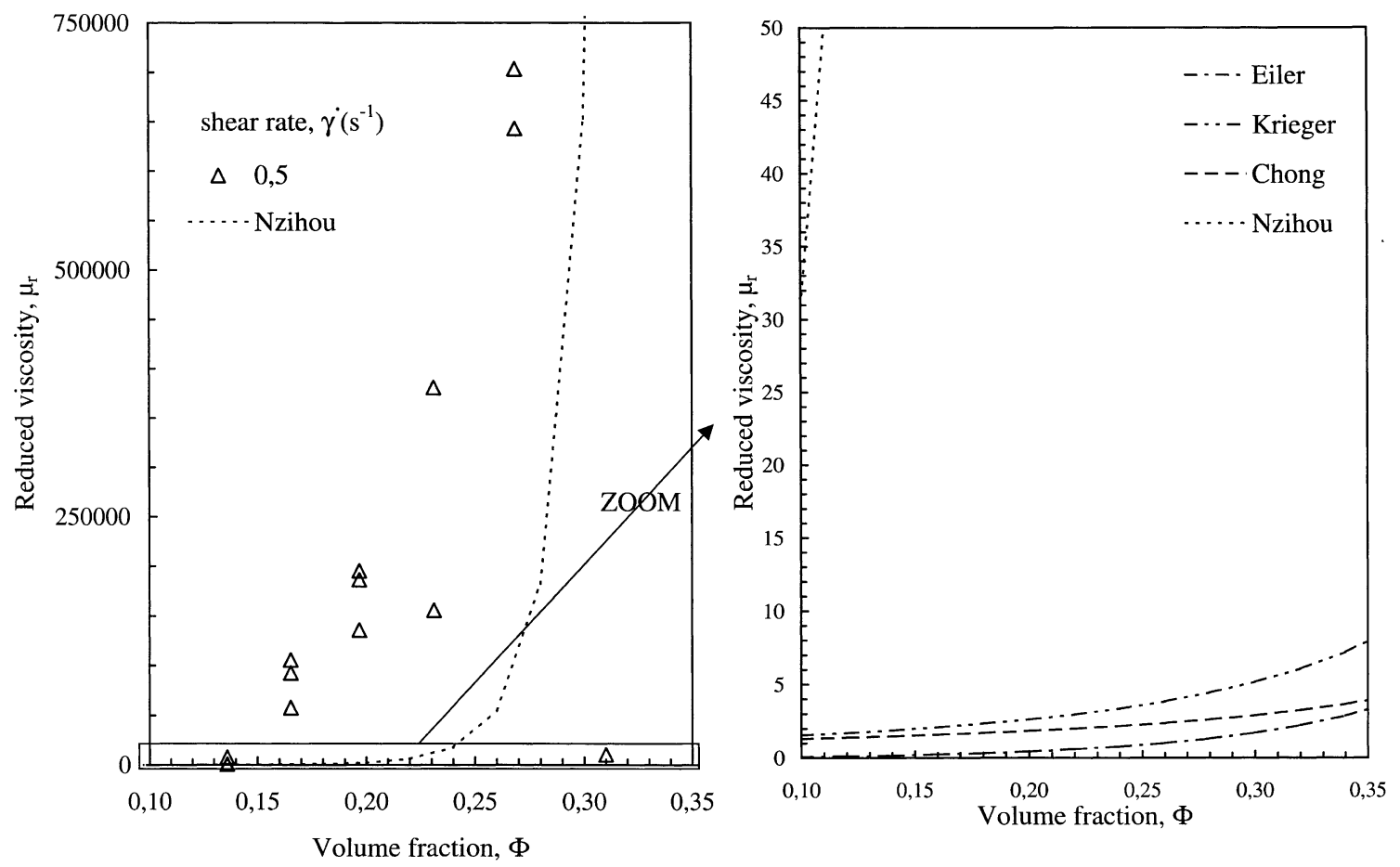

Fig. 8. Comparison between models and experimental data.

To improve this equation, three adjustable parameters were introduced in the empirical equation Nzihou et al. It then becomes:

$\mu_{\mathrm{r}}=\left[1+\frac{D}{\dot{\gamma}^{E}} \frac{\frac{\phi}{\phi_{\max }}}{\left(1-\frac{\phi}{\phi_{\max }}\right)}\right]^{G}$

The values of $D$ and $E$ and $G$ constants are represented in Table 2.

This approach takes into account both the shear rate and the solid volume fraction. The physical mean of the constants is found in the Krieger-Dougherty equation (Eq. (8)) in which the exponent is equal to 2.5 (parameter $D$ in Eq. (13)), the exponent of the shear rate $(E=0.5)$ is according to Casson model (Eq. (22)), which is used to describe yield stress materials with $D$ as a constant.

The relative viscosity $\mu_{\mathrm{r}}$ is plotted versus volume fraction of the two ashes in Fig. 9a and b. A good agreement is observed between experimental data and model (solid line). Fig. 9 reveals that the viscosity of the suspensions increases with increasing solid volume fraction as is usual for concentrated suspensions. We also observe that, at constant solid

Table 2

Values of $D$ and $E$ and $G$ constants of Eq. (13)

\begin{tabular}{llll}
\hline Constant & $D$ & $E$ & $G$ \\
\hline S fly ash & 160 & 0.5 & 2.5 \\
O fly ash & 170 & 0.5 & 2.5 \\
\hline
\end{tabular}

volume fraction, viscosity decreases with shear rate, causing a shear-thinning behaviour.

The decrease of viscosity with increasing shear rate can be attributed to a breakdown of the structure of the suspension. Particles will become more aligned and hence less entangled and less resistant to deformation as the deformation rate or shear rate is increased.

The next step in this work will be to investigate the rheological behaviour of fly ash suspensions during phosphate reaction to stabilize the heavy metals.

\subsection{Predominant effects}

Many effects can be taken into account when a suspension is sheared. The predominant ones are the Peclet number [24-26] and the Reynolds number.

The ratio between the viscous and Brownian effects is the Peclet number:

$P e=\frac{r^{2} \dot{\gamma} f}{k T}$

$f$ depends on the rheological parameter of the surrounding fluid; $r$ is the particle radius and $k T$, the usual unit of thermal energy.

The inertia and viscous effects expressed by the Reynolds number

$R e=\frac{\rho d u}{\mu_{\mathrm{e}}}$ 


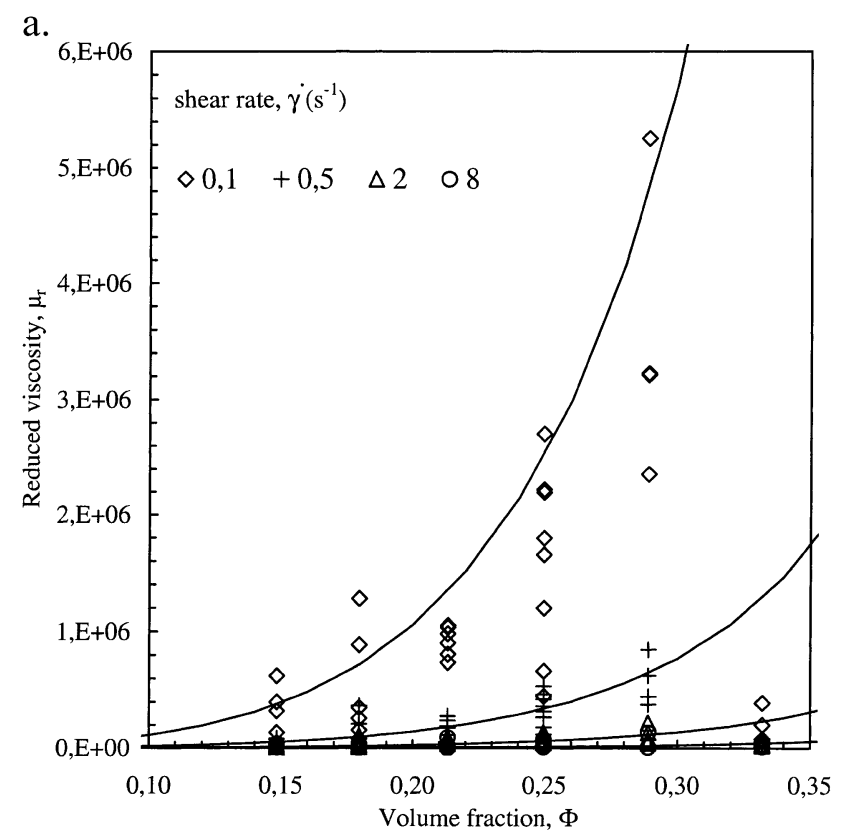

b.

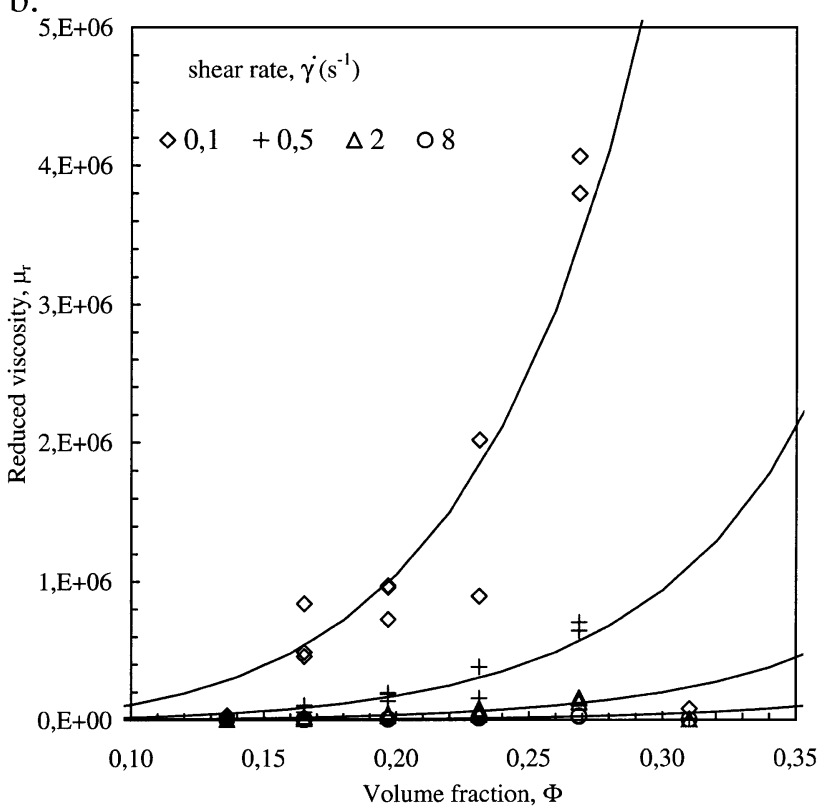

Fig. 9. (a) Comparison between models and experimental data of fly O. (b) Comparison between models and experimental data of fly $\mathrm{S}$.

When the hydrodynamic effects dominate at sufficiently low shear rates, the Reynolds number for non-Newtonian sheared system is defined as follows:

$\operatorname{Re}=\frac{\rho d^{2} \dot{\gamma}}{\mu_{e}}$

$\rho$ is the mean density of the suspension $\left(\mathrm{kg} / \mathrm{m}^{3}\right)$, defined by $\rho=\phi \rho_{\mathrm{p}}+(1-\phi) \rho_{\mathrm{s}}$, d the diameter of the stirring agitator and $\mu_{\mathrm{e}}$ the effective viscosity, which is a function of the volume fraction of solids.

To compare the hydrodynamic effect in the results obtained using the rheometer with those obtained in a stirred tank reactor, two definitions of viscosity were used for calculating the Reynolds number. We propose a first equation which expresses viscosity in terms of the volume fraction of solids and shear rate (Eq. (13) or (17)). The second approach matches the data using the Herschel-Bulkley approach (Eq. (20)), which takes into account the yield stress observed with the two fly ashes for the calculation of the Reynolds number and gives information about the predominant effects during shearing of the fly ash suspension. Several definitions of viscosity are used:

$\mu=\mu_{\mathrm{e}}=\mu_{\mathrm{s}}\left[1+\frac{D}{\dot{\gamma} E} \frac{\frac{\phi}{\phi_{\max }}}{\left(1-\frac{\phi}{\phi_{\max }}\right)}\right]^{G}$

The Reynolds number is then

$$
\operatorname{Re}=\frac{\rho d^{2} \dot{\gamma}}{\mu_{\mathrm{s}}\left[1+\frac{D}{\dot{\gamma}^{E}} \frac{\frac{\phi}{\phi_{\max }}}{\left(1-\frac{\phi}{\phi_{\max }}\right)}\right]^{G}}
$$

The measurement found with the rheometer was fitted by Herschel-Bulkley equation:

$\tau=\tau_{\mathrm{c}}+\mu \dot{\gamma}^{n}$

The viscosity is then defined by

$\mu=\frac{\tau-\tau_{\mathrm{c}}}{\dot{\gamma}^{n}}$

The Reynolds number is then defined by the equation:

$R e=\frac{d^{2} \rho \dot{\gamma}^{n+1}}{\tau-\tau_{\mathrm{c}}}$

The value of the consistency constant, $n$ was calculated using experimental results.

The behaviour of the suspension of fly ash can be described by the Casson equation [27,28]:

$\tau^{0.5}=\tau_{\mathrm{c}}^{0.5}+\left(\mu_{\mathrm{c}} \dot{\gamma}\right)^{0.5}$

This approach was also used in the literature [29] to describe the behaviour of dense suspensions. The corresponding apparent viscosity is

$\mu=\frac{\tau}{\dot{\gamma}}=\left[\mu_{\mathrm{c}}^{0.5}+\left(\frac{\tau_{\mathrm{c}}}{\dot{\gamma}}\right)^{0.5}\right]^{2}$

Thus, the Reynolds number for the suspension can be obtained by substituting Eq. (23) into (16).

$\operatorname{Re}=\frac{d u \rho}{\left[\mu_{\mathrm{c}}^{0.5}+\left(\frac{\tau_{\mathrm{c}}}{\dot{\gamma}}\right)^{0.5}\right]^{2}}=\frac{d^{2} \rho \dot{\gamma}}{\left[\mu_{\mathrm{c}}^{0.5}+\left(\frac{\tau_{\mathrm{c}}}{\dot{\gamma}}\right)^{0.5}\right]^{2}}$ 
In this study, only the Reynolds number which takes into account the volume fraction of solid in the suspension and the shear rate will be tested to better understand the hydrodynamics of particle movement in the shear-thinning suspension of fly ash. The main objective is to compare in the near future these results with those obtained in a stirred tank reactor.

The Reynolds number corresponding to the shear conditions in the rheometer for fly ash $\mathrm{S}$ at several solid volume fractions are presented in Figs. 10 and 11. The plots representing the $R e-\phi$ and $R e-\dot{\gamma}$ show a good agreement between the two approaches using different viscosity equations for the calculation of the Reynolds number. Nevertheless, only the results obtained with our approach (Eq. (18)) are presented.

With both Eqs. (18) and (21), the Reynolds number is much lesser than 30 in reference to the agitator in a nonNewtonian fluid [30-32]. It corresponds to the laminar flow regime of the suspension of fly ash in the domain investigated (volume fraction and shear rate, i.e. $\phi=0.13$ to $\phi=$ 0.30 and $\dot{\gamma}$ from 0.01 to $8 \mathrm{~s}^{-1}$ ).

For suspension with a yield stress, it is necessary to compare inertia effects to viscous effects due to colloidal interaction. In our experimental conditions, inertia effects cannot be neglected in comparison to hydrodynamic effects (viscous effects). The exact transition range for which inertia effects are significant depends on the boundary conditions and should be determined experimentally. For non-Newtonian fluids, and in particular for yield stress fluids, it is only apparent from existing data that the critical range of $\mathrm{Re}$ corresponding to the transition between laminar and a turbulent flow regime, has generally much larger boundary than for Newtonian fluids [33].

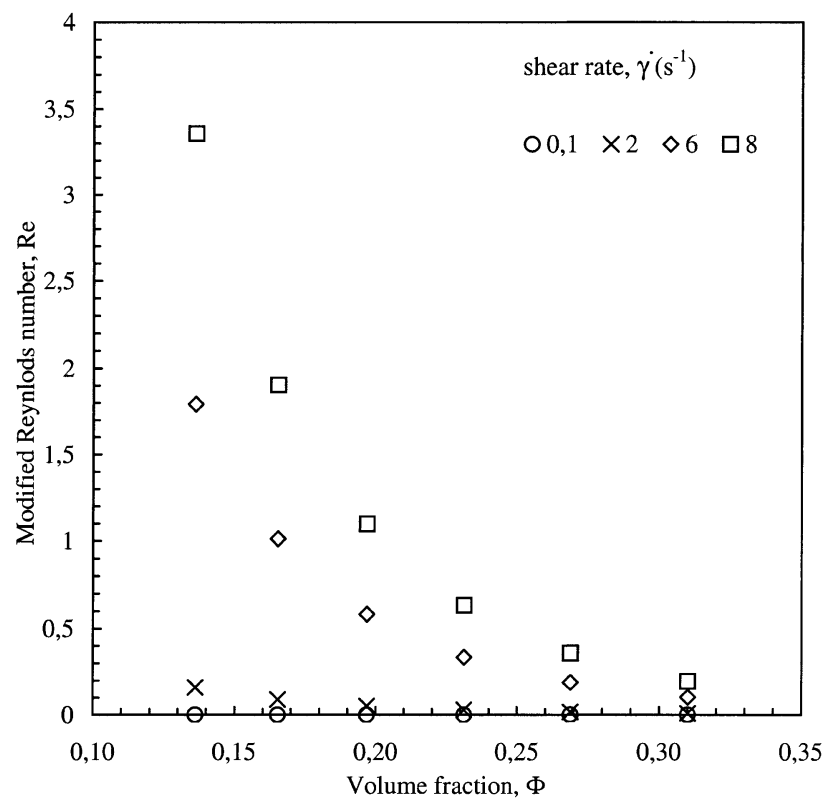

Fig. 10. Effects of volume fraction of solid on the flow behaviour for the fly ash $\mathrm{S}$ using Eq. (18).

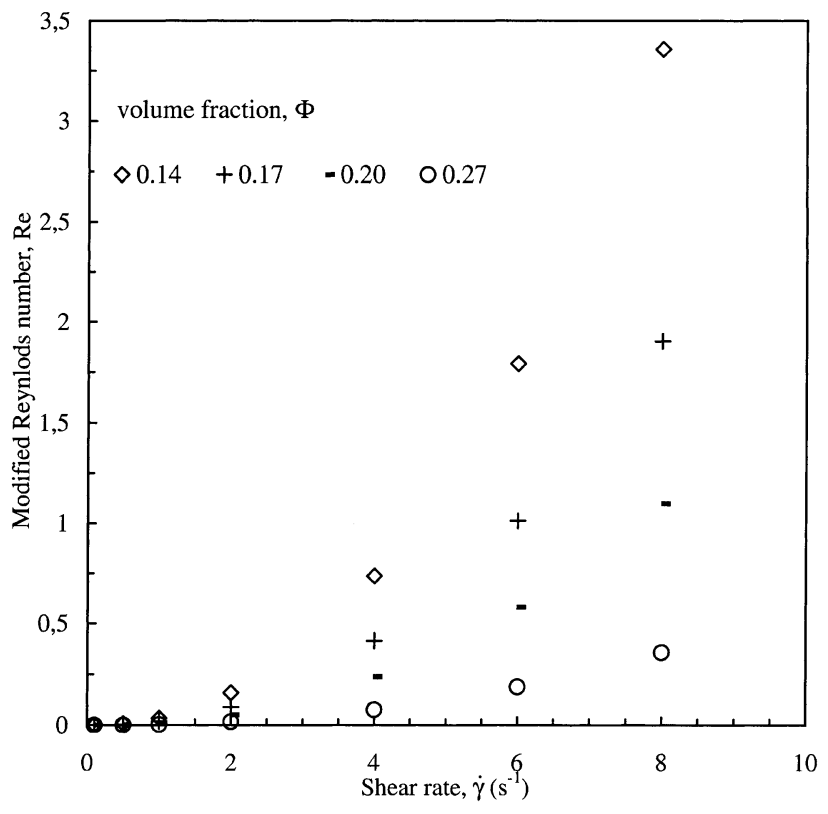

Fig. 11. Effects of shear rate on the flow behaviour for the fly ash $\mathrm{S}$ using Eq. (18).

The flow regime in the suspension of small particles like fly ash is well described [34]. The larger colloidal particles interact through interparticle colloidal forces, e.g. LondonVan der Waals, electrostatic, steric, etc., and through hydrodynamic forces transmitted through the fluid. In the case of sufficiently small particles (generally one micron or less), thermal fluctuation in the continuous phase gives rise to Brownian motion, which can also be related to the hydrodynamic interactions through the fluctuation-dissipation theorem. As long as the Reynolds number for the particle motion is small $(R e \ll 1)$, the hydrodynamic interactions are governed by the linearized Navier-Stokes equations, which are known simply as the Stokes equations. The final area is when the Reynolds number is neither small nor large. The solution of the Navier-Stokes equations determines the hydrodynamic forces [35].

\section{Conclusion}

Rheological experiments were carried out to better understand the stabilization process of heavy metals by phosphate reaction with two fly ashes.

A shear-thinning and thixotropic behaviour is observed for aqueous suspensions of non-treated fly ashes. The study shows that the viscosity is sensitive to the concentration of solids. A comprehensive model to predict the results, based on the concentrated suspension equation proposed by Chong et al. and Nzihou et al. was adapted and correlated to experimental data.

In order to describe the movement of solid particles in nonNewtonian fluids, the viscosity term in the general form of the Reynolds number equation was replaced by an apparent vis- 
cosity term, which takes into account the volume fraction of solids, the shear rate and the yield stress. This allows one to consider the non-Newtonian properties of the yield stress suspension in studying the movement of solid particles at the rheometer scale. Incinerator fly ash aqueous suspensions are well described by the proposed model (Eq. (13)) and by the Herschel-Bulkley model (Eq. (20)). Viscosity can then be calculated, and the Reynolds number derived, using these equations. It is concluded that the flow regime is laminar and inertial effects cannot be neglected.

In future work, the effect of temperature on the viscosity and rheological behaviour in aqueous suspension of phosphate-treated fly ash must be investigated. The model developed here will be used to extrapolate the behaviour observed in stirred tank reactor using the Metzner-Otto [36,37] approach which takes into account the geometry, the Reynolds number and power consumption of the stirred tank reactor, and will establish the correspondence between rheometer measurements and stirred tank reactors.

$\begin{array}{ll}\text { Nomenclature } \\ A & \text { constant } \\ B & \text { constant } \\ d & \text { diameter of the plate in the rheometer } \\ D & \text { constant, } \mathrm{s}-0,5 \\ E & \text { constant } \\ \Delta F & \text { applied force, } \mathrm{N} \\ f & \text { parameter function of surrounding fluid } \\ G & \text { constant } \\ K & \text { constant, } \mathrm{s} \\ k & \text { Boltzmann constant } \\ m & \text { consistency in Cross model } \\ n & \text { consistency in Herschel-Bulkey model } \\ P e & \text { Peclet number } \\ r & \text { particle radius, } \mathrm{m} \\ R e & \text { Reynolds number } \\ \Delta S & \text { surface, }{ }^{2} \\ t & \text { time, } \mathrm{s} \\ T & \text { temperature, } \mathrm{K} \\ u & \text { flow velocity, m s } \\ V & \text { volume of the suspension, } \mathrm{m}^{3} \\ V_{\mathrm{p}} & \text { fly ash particle volume, } \mathrm{m}^{3} \\ V_{\mathrm{s}} & \text { continuous phase volume, } \mathrm{m}^{3} \\ X & \text { solid mass fraction, } \% \\ & \end{array}$

$\begin{array}{ll}\rho_{\mathrm{p}} & \text { fly ash particle density, } \mathrm{kg} \mathrm{m}^{-3} \\ \rho_{\mathrm{s}} & \text { continuous phase (water) density, } \mathrm{kg} \mathrm{m}^{-3} \\ \phi & \text { volume fraction } \\ \phi_{\max } & \text { maximal volume fraction } \\ \tau & \text { shear stress, } \mathrm{Pa} \\ \tau_{\mathrm{c}} & \text { yield stress, Pa } \\ & \\ \text { Subscript } \\ \mathrm{c} & \text { yield } \\ \mathrm{e} & \text { effective } \\ \text { max } & \text { maximal } \\ 0 & \text { initial } \\ \mathrm{p} & \text { particle } \\ \mathrm{r} & \text { reduced } \\ \mathrm{S} & \text { continuous } \\ \infty & \text { infinite }\end{array}$

\section{Acknowledgements}

These investigations were supported with financial assistance from the SOLVAY Group. This support is gratefully acknowledged.

\section{References}

[1] R. Derie, Waste Manage. 16 (1996) 711.

[2] T.T. Eighmy, B.S. Crannell, F.K. Cartledge, E.F. Emery, D. Oblas J.E. Krzanowski, E.L. Shaw, C.A. Francis, Environ. Sci. Technol. 31 (1997) 3330

[3] S. Iretskaya, A. Nzihou, C. Zahroui, P. Sharrock, Environ. Prog. 18 (1999) 144.

[4] H.A. Barnes, J.F. Hutton, K. Walters, in: Elsevier (Eds.), An Introduction to Rheology, Rheology Series, Amsterdam, 1998.

[5] M. Perez, J.-C. Barbé, Z. Neda, Y. Bréchet, L. Salvo, Acta Mater. 48 (2000) 3773.

[6] J. Mewis, J. Non-Newton. Fluid Mech. 6 (1979) 1.

[7] J. Mewis, Cah. Rhéol. (French Rheology Group) XIII (1994) 2

[8] E. Ruckenstein, J. Mewis, J. Colloid Interface Sci. 44 (1973) 532.

[9] L.L. Navickis, E.B. Bagley, J. Rheol. 27 (1983) 519.

[10] P. Coussot, A.I. Leonov, J.-M. Piau, J. Non-Newton. Fluid Mech. 46 (1993) 179.

[11] W.B. Russel, Annu. Rev. Fluid Mech. 13 (1981) 425.

[12] J. Mewis, A.J.B. Spaull, Adv. Colloid Interface Sci. 6 (1976) 173.

[13] H.K. Kytomaa, D. Prasad, in: C. Thornton (Ed.), Transition from Quasi-Static to Rate Dependent Shearing of Concentrated Suspensions, Powders \& Grains, Balkema, Rotterdam, 1993, p. 281.

[14] D.M. Husband, N. Aksel, W. Gleissle, J. Rheol. 37 (1993) 215

[15] P. Coussot, J.-M. Piau, Cah. Rhéol. (Groupe Français de Rhéologie) XIII (1994) 266.

[16] O. Reynolds, Philos. Mag. J. Sci., Ser. 5 (20) (1885) 469.

[17] D. Quemada, J. Mec. Theor. Appl., (1985) 267.

[18] D.J. Jeffrey, A. Acrivos, AIChE J. 22 (1976) 417.

[19] V.V. Jinescu, Int. Chem. Eng. 14 (1974) 397.

[20] H. Eiler, Kolloid-Z. 102 (1943) 154.

[21] J.S. Chong, E.B. Christiansen, A.D. Baer, J. Appl. Polym. Sci. 15 (1971) 2007

[22] I.M. Krieger, T.J. Dougherty, Trans. Soc. Rheol. 3 (1959) 137.

[23] A. Nzihou, L. Attias, P. Sharrock, A. Ricard, Powder Technol. 99 (1998) 60 . 
[24] I.M. Krieger, Trans. Soc. Rheol. 7 (1963) 101.

[25] I.M. Krieger, Adv. Colloid Interface Sci. 3 (1972) 111

[26] Z. Zhou, P.J. Scales, D.V. Boger, Chem. Eng. Sci. 56 (2001) 2901

[27] B. Klein, J.S. Laskowski, S.J. Partridge, J. Rheol. 39 (1995) 827.

[28] Y.B. He, J.S. Laskowski, Miner. Process. Extr. Metall. Rev. 20 1999, p. 167.

[29] Y.B. He, Laskowski, B. Klein, Chem. Eng. Sci. 56 (2001) 2991.

[30] P.J. Carreau, R.P. Chhabra, J. Cheng, AIChE J. 39 (1993) 1421.
[31] L. Choplin, Ph. Marchal, Ann. Trans. Nord. Rheol. Soc. 7 (1999) 5.

[32] F. Delplace, J.C. Leuliet, Chem. Eng. J. 56 (1995) 33.

[33] N. Midoux, Mécanique et Rhéologie des Fluides en Génie Chimique, Tec \& Doc, Lavoisier, Paris, 1993

[34] J.F. Brady, Chem. Eng. Sci. 56 (2001) 2921.

[35] G. Bossis, J.F. Brady, J. Colloid Sci. 126 (1988) 1

[36] A.B. Metzner, R.E. Otto, AIChE J. 3 (1957) 3.

[37] A.W. Nienow, T.P. Elson, Chem. Eng. Res. Des. 66 (1988) 5. 